\title{
Trefftz discontinuous Galerkin methods for acoustic scattering on locally refined meshes
}

Article

Accepted Version

Hiptmair, R., Moiola, A. and Perugia, I. (2013) Trefftz discontinuous Galerkin methods for acoustic scattering on locally refined meshes. Applied Numerical Mathematics, 79. pp. 79-91. ISSN 0168-9274 doi: https://doi.org/10.1016/j.apnum.2012.12.004 Available at https://centaur.reading.ac.uk/30658/

It is advisable to refer to the publisher's version if you intend to cite from the work. See Guidance on citing.

Published version at: http://www.sciencedirect.com/science/article/pii/S0168927413000044

To link to this article DOI: http://dx.doi.org/10.1016/j.apnum.2012.12.004

Publisher: Elsevier

All outputs in CentAUR are protected by Intellectual Property Rights law, including copyright law. Copyright and IPR is retained by the creators or other copyright holders. Terms and conditions for use of this material are defined in the End User Agreement.

www.reading.ac.uk/centaur 
Central Archive at the University of Reading

Reading's research outputs online 


\section{Accepted Manuscript}

Trefftz discontinuous Galerkin methods for acoustic scattering on locally refined meshes

Ralf Hiptmair, Andrea Moiola, Ilaria Perugia

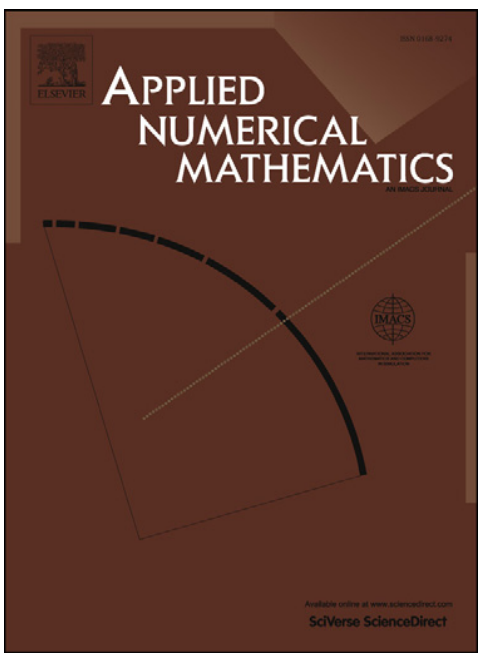

PII: $\quad$ S0168-9274(13)00004-4

DOI: $\quad$ 10.1016/j.apnum.2012.12.004

Reference: $\quad$ APNUM 2691

To appear in: Applied Numerical Mathematics

Received date: 3 April 2012

Revised date: 10 October 2012

Accepted date: 18 December 2012

Please cite this article in press as: R. Hiptmair et al., Trefftz discontinuous Galerkin methods for acoustic scattering on locally refined meshes, Applied Numerical Mathematics (2013), http://dx.doi.org/10.1016/j.apnum.2012.12.004

This is a PDF file of an unedited manuscript that has been accepted for publication. As a service to our customers we are providing this early version of the manuscript. The manuscript will undergo copyediting, typesetting, and review of the resulting proof before it is published in its final form. Please note that during the production process errors may be discovered which could affect the content, and all legal disclaimers that apply to the journal pertain. 


\title{
Trefftz discontinuous Galerkin methods for acoustic scattering on locally refined meshes
}

\author{
Ralf Hiptmair ${ }^{1}$, Andrea Moiola ${ }^{2}$, Ilaria Perugia ${ }^{3}$
}

\begin{abstract}
We extend the a priori error analysis of Trefftz-discontinuous Galerkin methods for time-harmonic wave propagation problems developed in previous papers to acoustic scattering problems and locally refined meshes. To this aim, we prove refined regularity and stability results with explicit dependence of the stability constant on the wave number for non convex domains with non connected boundaries. Moreover, we devise a new choice of numerical flux parameters for which we can prove $L^{2}$-error estimates in the case of locally refined meshes near the scatterer. This is the setting needed to develop a complete $h p$-convergence analysis.
\end{abstract}

Keywords: Acoustic scattering, wave propagation, discontinuous Galerkin methods, Trefftz methods, $h p$-error analysis, duality estimates

65N15, 65N30, 35J05

\section{Introduction}

Trefftz-discontinuous Galerkin methods (TDG) are volume mesh based discretizations of linear boundary value problems that aim to achieve effi-

\footnotetext{
Email addresses: hiptmair@sam.math.ethz.ch (Ralf Hiptmair), a.moiola@reading.ac.uk (Andrea Moiola), ilaria.perugia@unipv.it (Ilaria Perugia)

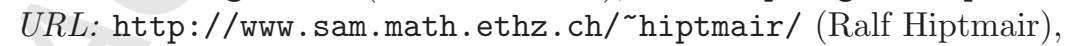
http://www.reading.ac.uk/maths-and-stats/about/Staff/a-moiola.aspx (Andrea Moiola), http://www-dimat.unipv.it/perugia/ (Ilaria Perugia)

${ }^{1}$ Seminar für Angewandte Mathematik ETHZ, Rämistrasse 101, 8092 Zürich, Switzerland

${ }^{2}$ Department of Mathematics and Statistics, University of Reading, Whiteknights, PO Box 220, Reading RG6 6AX, UK

${ }^{3}$ Dipartimento di Matematica, Università di Pavia Via Ferrata 1, 27100 Pavia, Italy
} 
cient approximation by the use of trial functions in the null spaces of the differential operator. They have attracted particular attention for problems that are notoriously challenging for polynomial approximation. A prominent representative is time-harmonic acoustic wave propagation in the medium frequency range, whose TDG discretization is at the focus of this article.

The method itself in combination with plane wave approximation was introduced in [14] and extended to electromagnetic fields in [18]. It generalizes the so-called ultra-weak variational formulation (UWVF) by Cessenat and Després [8, 7]. Practical experience with this method [19] suggests that for good performance it should be used on locally refined meshes together with spatially varying resolution of the plane wave trial space. Morally speaking, the sophisticated $h p$-refinement strategy that ensures exponential convergence (in the number of degrees of freedom) for classical polynomial Galerkin finite element approximation of second-order elliptic boundary value problem (see [2, 36, 34, 35]; see also [24] for the Helmholtz problem) should also be adopted for TDG.

Hitherto, in the DG context, only polynomial theory could cover this setting [13, 23], but it remained outside the scope of existing TDG theory. Whereas the a priori convergence estimates in [14] merely addressed $h$-refinement, the corresponding $p$-convergence results in [16] exclude local mesh refinement and require convex computational domains. The present article remedies these shortcomings and establishes TDG discretization error estimates for acoustic scattering at a sound-soft object, which remain valid for arbitrary variable resolutions of the local Trefftz trial spaces and in the presence of rather general local mesh refinement. More precisely, quasiuniformity must hold only close to the outer (smooth) artificial boundary, while strong local refinement near the scatterer is allowed, see Figure 1. All meshes created by standard $h p$-refinement policies belong to this class.

The new theory is a substantially enhanced version of the approach of [16] (see also [25, Sec. 4.3]). Again we employ a duality argument similar to the ones used in $[29,5]$. Yet, in order to dispense with the convexity assumption, we prove refined regularity and stability results for the acoustic scattering problem with explicit dependence of the stability constant on the wave number, see Sect. 2.1. These estimates are combined with the key idea to offset non-uniform element sizes by judiciously varying local parameters in the numerical fluxes for the discontinuous Galerkin discretization, see Sect. 4.2. This paves the way for the main $L^{2}$-error estimate of Theorem 4.5.

The results of this paper are a stepping stone towards a complete $h p$ - 


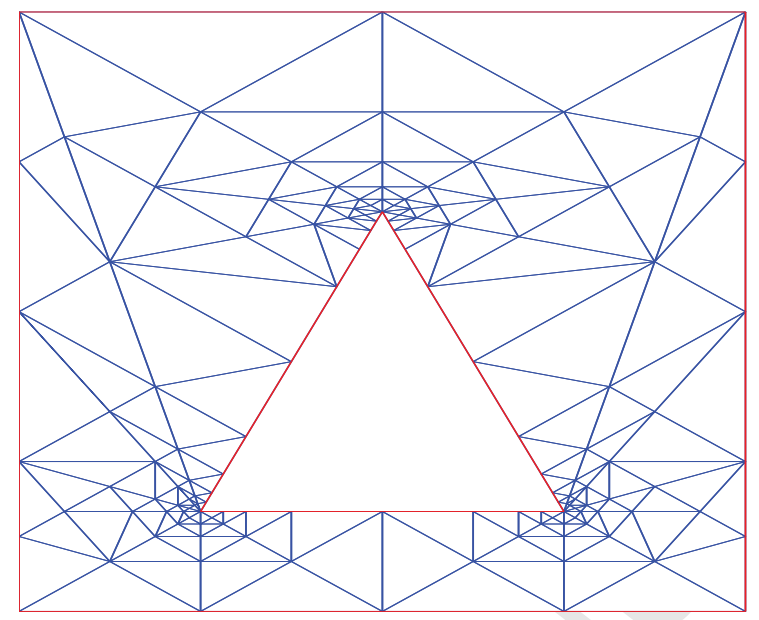

Figure 1: Mesh locally refined near the scatterer

convergence theory that we hope will eventually confirm exponential convergence (in terms of number of degrees of freedom) of a wide range of TDG methods for the Helmholtz equation, provided proper local refinements and choice of local resolutions. As of 2012, this is ongoing work and preliminary results are reported in Sect. 5 .

\section{Scattering problem}

We consider the scattering of acoustic waves at a sound soft scatterer occupying the domain $\Omega_{D} \subset \mathbb{R}^{N}, N=2,3$. This domain is supposed to be a bounded Lipschitz polygon $(N=2)$ or polyhedron $(N=3)$ that is starshaped with respect to the origin $\mathbf{0}$. The medium outside $\Omega_{D}$ is supposed to be homogeneous and isotropic.

A known time-harmonic smooth incident field impinges on $\Omega_{D}$. It is described by its complex amplitude $u^{i}$ and wave number $k=\omega / c$, where $\omega$ denotes the angular frequency and $c$ the speed of sound in the medium. To fix the notation, we assume $k>0$, although the sign of $k$ is not essential.

The total field $u=u^{i}+u^{s}$, where $u^{s}$ is the scattered wave, satisfies the following Dirichlet boundary value problem for the Helmholtz equation in 
$\mathbb{R}^{N} \backslash \bar{\Omega}_{D}($ see $[$ Sect. 2.1][10]):

$$
\begin{array}{r}
-\Delta u-k^{2} u=0 \quad \text { in } \mathbb{R}^{N} \backslash \bar{\Omega}_{D} \\
u=0 \quad \text { on } \Gamma_{D}:=\partial \Omega_{D} \\
\lim _{|\mathbf{x}| \rightarrow \infty}|\mathbf{x}|\left(\frac{\partial u^{s}(\mathbf{x})}{\partial|\mathbf{x}|}+i k u^{s}(\mathbf{x})\right)=0
\end{array}
$$

The use of volume mesh based discretization entails truncation of the unbounded domain. Therefore, we introduce another bounded Lipschitz domain $\Omega_{R}$, with boundary $\Gamma_{R}$, that contains $\bar{\Omega}_{D}$ such that

$$
\operatorname{dist}\left(\Gamma_{D}, \Gamma_{R}\right)>0
$$

see Fig. 2. The outer boundary $\Gamma_{R}$ will usually be smooth, though we also admit polygonal $(N=2)$ or polyhedral $(N=3) \Omega_{R}$. The last condition

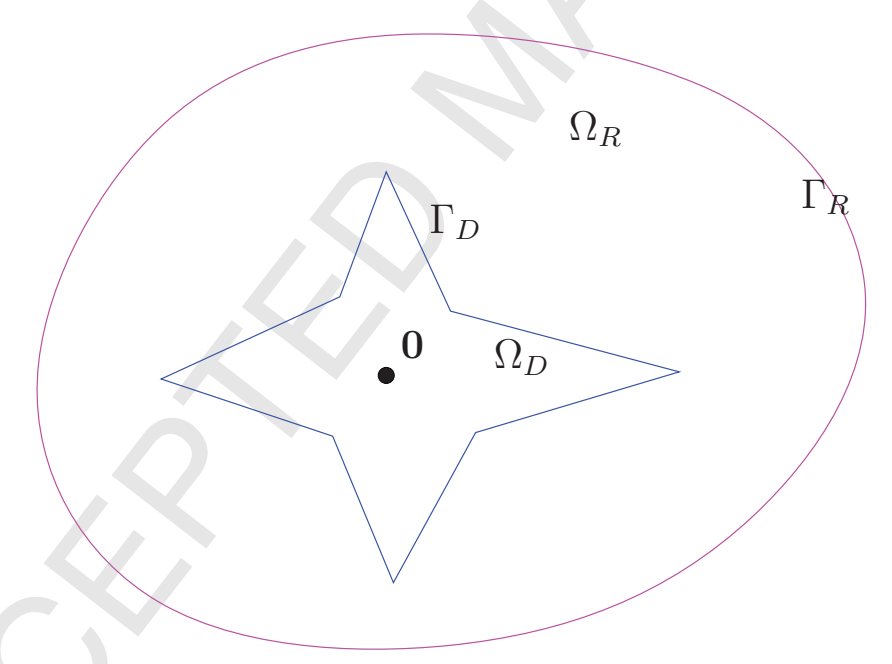

Figure 2: Geometric setting for boundary value problem

in (1) (i.e., the Sommerfeld radiation condition) can be approximated by an impedance boundary condition as a first order absorbing boundary condition:

$$
\begin{aligned}
-\Delta u-k^{2} u & =0 & & \text { in } \Omega:=\Omega_{R} \backslash \bar{\Omega}_{D}, \\
u & =0 & & \text { on } \Gamma_{D}, \\
\frac{\partial u}{\partial \mathbf{n}}+i k \vartheta u & =g_{R} & & \text { on } \Gamma_{R} .
\end{aligned}
$$


Here, $\mathbf{n}$ is the unit normal vector field that is outgoing from $\Omega:=\Omega_{R} \backslash \bar{\Omega}_{D}$ (i.e., outgoing from $\Omega_{R}$ and ingoing into $\Omega_{D}$ ), and $\vartheta$ is the (non-dimensional) impedance of the exterior medium. Usually, $\vartheta=1$, but, for the sake of generality, we admit real valued functions $\vartheta: \Gamma_{R} \rightarrow \mathbb{R}$ such that,

$$
0<\vartheta_{*}:=\inf _{\mathbf{x} \in \Gamma_{R}} \vartheta(\mathbf{x}) \leq \sup _{\mathbf{x} \in \Gamma_{R}} \vartheta(\mathbf{x})=: \vartheta^{*}<\infty
$$

Moreover, $g_{R}:=\frac{\partial u^{i}}{\partial \mathbf{n}}+i k \vartheta u^{i}$ is the impedance trace of the incoming wave, and, thus, it is supposed to be known data.

We assume that the artificial domain $\Omega_{R}$ is star-shaped with respect to the open ball $B_{\gamma_{R} d_{\Omega}}(\mathbf{0})=\left\{\mathbf{x} \in \mathbb{R}^{N},|\mathbf{x}|<\gamma_{R} d_{\Omega}\right\}$, for some $\gamma_{R}>0$, where

$$
d_{\Omega}:=\operatorname{diam}(\Omega)
$$

this and the assumption that the scatterer $\Omega_{D}$ is star-shaped with respect the origin translate into

$$
\mathbf{n}(\mathbf{x}) \cdot \mathbf{x} \leq 0 \text { a.e. on } \Gamma_{D}, \quad \mathbf{n}(\mathbf{x}) \cdot \mathbf{x} \geq \gamma_{R} d_{\Omega}>0 \text { a.e. on } \Gamma_{R} .
$$

These inequalities are meant to hold for every point $\mathbf{x}$ at which $\mathbf{n}(\mathbf{x})$ is defined (see [17, Lemma 3.1]).

We point out that our approach can also deal with the case $\Gamma_{D}=\emptyset$, i.e., a cavity impedance boundary value problem without scatterer.

Notice that this setting is different from the one studied in [9], where the domains are assumed to be smooth, and the exact Dirichlet-to-Neumann map is used instead of the impedance boundary condition.

\subsection{Well-posedness and stability}

For later use in a duality argument, we consider a slightly more general situation than (2), namely the inhomogeneous boundary value problem:

$$
\begin{aligned}
-\Delta u-k^{2} u & =f & & \text { in } \Omega, \\
u & =0 & & \text { on } \Gamma_{D}, \\
\frac{\partial u}{\partial \mathbf{n}} \pm i k \vartheta u & =g_{R} & & \text { on } \Gamma_{R},
\end{aligned}
$$

for given $f \in L^{2}(\Omega)$ and $g_{R} \in L^{2}\left(\Gamma_{R}\right)$.

We introduce the space $H_{\Gamma_{D}}^{1}(\Omega)=\left\{u \in H^{1}(\Omega), u=0\right.$ on $\left.\Gamma_{D}\right\}$. We denote by $\|\cdot\|_{s, D}$ the $H^{s}(D)$-norm, $s \in \mathbb{R}$, and by $|\cdot|_{\ell, D}$ the $H^{\ell}(D)$-seminorm, 
$\ell \in \mathbb{N}$, where $D$ is either a domain or a manifold (of smoothness $C^{\lceil|s|\rceil-1,1}$ and $C^{\ell-1,1}$, respectively). We also define the following $k$-weighted Sobolev norms on $D$ :

$$
\|v\|_{\ell, k, D}^{2}:=\sum_{j=0}^{\ell} k^{2(\ell-j)}|u|_{j, D}^{2} \quad \forall v \in H^{\ell}(D), \ell \in \mathbb{N} .
$$

The variational formulation of the boundary value problem (3) reads: find $u \in H_{\Gamma_{D}}^{1}(\Omega)$ such that, for all $v \in H_{\Gamma_{D}}^{1}(\Omega)$,

$$
\int_{\Omega}\left(\nabla u \cdot \nabla \bar{v}-k^{2} u \bar{v}\right) \mathrm{d} V \pm i k \int_{\Gamma_{R}} \vartheta u \bar{v} \mathrm{~d} S=\int_{\Omega} f \bar{v} \mathrm{~d} V+\int_{\Gamma_{R}} g_{R} \bar{v} \mathrm{~d} S .
$$

The use of impedance (Robin) boundary condition prevents the occurrence of eigenvalues and ensures the well-posedness of the problem.

Theorem 2.1. Problem (4) admits a unique solution $u \in H_{\Gamma_{D}}^{1}(\Omega)$.

Proof. The bilinear form on the right-hand side of (4) satisfies a Gårding inequality (see [20, p. 118]); then, by [20, Th. 4.11 and Th. 4.12], problem (4) (and thus (3)) admits a unique solution if and only if its adjoint problem with $f=0$ and zero boundary data $g_{R}=0$ admits only the trivial solution. Since the adjoint problem is obtained by simply switching the sign in front of the term $i k \vartheta u$ in the impedance boundary condition, we only have to show that, whenever $f=0$ and $g_{R}=0$, problem (3) has only the solution $u \equiv 0$.

In fact, if $f=0$ and $g_{R}=0$, by taking $v=u$ in (4), and considering the imaginary part of the resulting equation, we infer $\left\|\vartheta^{\frac{1}{2}} u\right\|_{0, \Gamma_{R}}=0$; thus, from the impedance boundary condition in (3), $\frac{\partial u}{\partial \mathbf{n}}=u=0$ on $\Gamma_{R}$. As a consequence, extending $u$ by zero outside $\Omega_{R}$ we obtain a function $\widetilde{u}$ that satisfies $-\Delta \widetilde{u}-k^{2} \widetilde{u}=0$ in $\mathbb{R}^{N} \backslash \bar{\Omega}_{D}$ and $\widetilde{u}=0$ on $\Gamma_{D}$. Owing to the uniqueness of solutions of exterior Dirichlet problems for the Helmholtz equation [31, Th. 2.6.5] we conclude $\widetilde{u}=0$.

The Fredholm argument in the proof of Theorem 2.1 also provides stability bounds on the solution $u$ of problem (4) in terms of the data $f$ and $g_{R}$, but with no information on the possible dependence of the stability constants on the wave number $k$. Since we need to know this dependence explicitly we rely on the stability results of [15], which were obtained by means of Rellich estimates (the same results were proved in [21, Prop. 8.1.4] in 2D, and in 
[11] in 3D in the case $\Gamma_{D}=\emptyset$; see also [33], [30, Chap. 5], [20, p. 146], [9, Lemma 2.3, Lemma 3.5], and [37]).

Theorem 2.2. [15, Prop. 3.3 and 3.4] Let u be the unique solution to problem (4). Under our assumptions on the problem domain and boundary conditions, there exists a constant $C>0$ depending only on $\gamma_{R}$ and $\vartheta$, but independent of $k, f, g_{R}$ and $u$, such that

$$
\|u\|_{1, k, \Omega} \leq C\left(d_{\Omega}\|f\|_{0, \Omega}+d_{\Omega}^{\frac{1}{2}}\left\|g_{R}\right\|_{0, \Gamma_{R}}\right) .
$$

We point out that the assumptions made in [15, Prop. 3.3 and 3.4] rule out the case of a sound hard scatterer (in the proofs of [15], a possible Neumann boundary $\Gamma_{N}$ has to be such that $\mathbf{n}(\mathbf{x}) \cdot \mathbf{x}=0$ on $\Gamma_{N}$, thus it can not be the boundary of a bounded region); for this reason we restrict ourselves to the case of a sound soft scatterer.

\subsection{Regularity}

Our error analysis of the TDG method hinges on the assumption of $H^{\frac{3}{2}+s_{-}}$ regularity of the analytical solutions of (3), for some positive $s$. The following theorem ensures this regularity and states stability estimates of the solutions in the $H^{\frac{3}{2}+s}$-norm.

Theorem 2.3. Let $u$ be the solution of problem (3). If $f \in L^{2}(\Omega)$ and $g_{R} \in H^{r}\left(\Gamma_{R}\right)$ for a given $0<r<1 / 2$, then there exists $s_{\Omega}>0$ depending only on (the edges and corners of) $\Omega$, such that $u \in H^{\frac{3}{2}+s}(\Omega)$ for every $s$ satisfying

$$
0<s<s_{\Omega}, \quad s \leq r,
$$

and the following bound holds ${ }^{4}$.

$$
|\nabla u|_{\frac{1}{2}+s, \Omega} \leq C\left(1+d_{\Omega} k\right)\left(d_{\Omega}^{\frac{1}{2}-s}\|f\|_{0, \Omega}+d_{\Omega}^{-s}\left\|g_{R}\right\|_{0, \Gamma_{R}}\right)+C\left\|g_{R}\right\|_{s, \Gamma_{R}},
$$

where the constant $C>0$ depends only on $s, \gamma_{R}$ and $\vartheta$, but is independent of $k, f, g_{R}$ and $u$.

\footnotetext{
${ }^{4}$ In order to obtain bounding constants that are independent of the size of $\Omega$, the fractional Sobolev norm are weighted with the diameter $d_{\Omega}$ as $\|v\|_{s, \Gamma_{R}}^{2}:=|v|_{s, \Gamma_{R}}^{2}+d_{\Omega}^{-2 s}\|v\|_{0, \Gamma_{R}}^{2}$, for every $v \in H^{s}\left(\Gamma_{R}\right)$ and $0<s<1 / 2$, where the $H^{s}$-seminorm is defined by the SobolevSlobodeckij integral (see [28, p. 43]).
} 
Proof. First, we consider the case $\Omega_{D}=\emptyset$. The second part of the NeumannPoisson regularity Theorem 3.18 of [28] tells us that there exists $s_{N}>0$ depending only on $\Omega$ such that $u$ belongs to $H^{\frac{3}{2}+s}(\Omega)$ for all $0<s<s_{N}$, $s \leq r$.

Moreover, define

$$
\begin{aligned}
& X=\left\{v \in H^{\frac{3}{2}+s}(\Omega): \Delta v \in L^{2}(\Omega), \int_{\Omega} v \mathrm{~d} V=0\right\} \\
& Y=\left\{(F, G) \in L^{2}(\Omega) \times H^{s}\left(\Gamma_{R}\right): \int_{\Omega} F \mathrm{~d} V+\int_{\Gamma_{R}} G \mathrm{~d} S=0\right\},
\end{aligned}
$$

and endow $X$ with the graph norm. The operator $L: X \rightarrow Y$ which maps $u \mapsto\left(\Delta u, \frac{\partial u}{\partial n}\right)$ is injective, by uniqueness of the solution of the NeumannPoisson problem in the subspace of $H^{1}(\Omega)$ of zero mean value functions, is surjective, by the above mentioned [28, Theorem 3.18, second part], and is continuous, by definition of the norm in $X$ and the trace theorem. By the open mapping theorem $L^{-1}$ is continuous. Thus, we have

$$
\begin{aligned}
|u|_{\frac{3}{2}+s, \Omega} & \leq C\left(d_{\Omega}^{\frac{1}{2}-s}\|\Delta u\|_{0, \Omega}+\left\|\frac{\partial u}{\partial \mathbf{n}}\right\|_{s, \Gamma_{R}}\right) \\
& \stackrel{(3)}{\leq} C\left(d_{\Omega}^{\frac{1}{2}-s}\|f\|_{0, \Omega}+d_{\Omega}^{\frac{1}{2}-s} k^{2}\|u\|_{0, \Omega}+\left\|g_{R}\right\|_{s, \Gamma_{R}}+k\|\vartheta\|_{L^{\infty}\left(\Gamma_{R}\right)}\|u\|_{s, \Gamma_{R}}\right) \\
\text { trace th. } & \quad \stackrel{\text { Th. 3.37] }}{\leq} C\left(d_{\Omega}^{\frac{1}{2}-s} k\|u\|_{1, k, \Omega}+d_{\Omega}^{\frac{1}{2}-s}\|f\|_{0, \Omega}+\left\|g_{R}\right\|_{s, \Gamma_{R}}\right) \\
& \quad \text { (5) } \\
\quad & C\left(1+d_{\Omega} k\right)\left(d_{\Omega}^{\frac{1}{2}-s}\|f\|_{0, \Omega}+d_{\Omega}^{-s}\left\|g_{R}\right\|_{0, \Gamma_{R}}\right)+C\left\|g_{R}\right\|_{s, \Gamma_{R}}
\end{aligned}
$$

where the constant $C>0$ is independent of $k$ and $u$.

Now we consider the case $\Omega_{D} \neq \emptyset$. Let $\partial G_{1}$ and $\partial G_{2}$ be two smooth closed curves/surfaces bounding the open domains $G_{1}$ and $G_{2}$, respectively, such that

$$
\bar{\Omega}_{D} \subset G_{1}, \quad \bar{G}_{1} \subset G_{2}, \quad \bar{G}_{2} \subset \Omega_{R} .
$$

Let $\chi \in \mathcal{D}\left(G_{2}\right)$ be a real-valued cut-off function such that $0 \leq \chi \leq 1$ and $\chi \equiv 1$ in $G_{1}$. Again, denote by $\chi$ its extension by zero to $\mathbb{R}^{N}$.

By the product rule $(\Delta(\phi \psi)=\phi \Delta \psi+\psi \Delta \phi+2 \nabla \phi \cdot \nabla \psi), u_{1}:=\chi u$ solves

$$
\begin{aligned}
-\Delta u_{1} & =\chi\left(f+k^{2} u\right)-u \Delta \chi-2 \nabla \chi \cdot \nabla u & & \text { in } \Omega, \\
u_{1} & =0 & & \text { on } \Gamma_{R} \cup \Gamma_{D} .
\end{aligned}
$$


The first part of Dirichlet-Poisson regularity Theorem 3.18 of [28] applied to the case of homogeneous Dirichlet boundary datum tells us that there exists $s_{D}>0$ depending only on $\Omega$ such that $u_{1} \in H^{\frac{3}{2}+s}(\Omega)$ for all $0<s<s_{D}$.

Moreover, define $X=\left\{v \in H^{\frac{3}{2}+s}(\Omega) \cap H_{0}^{1}(\Omega): \Delta v \in L^{2}(\Omega)\right\}$, and endow it with the graph norm. The operator $L: X \rightarrow L^{2}(\Omega)$ which maps $u \mapsto \Delta u$ is injective, by uniqueness of the solution of the Dirichlet-Poisson problem in $H^{1}(\Omega)$, is surjective, by the above mentioned [28, Theorem 3.18, first part], and is continuous, by definition of the norm in $X$. By the open mapping theorem $L^{-1}$ is continuous. Therefore, we infer

$$
\begin{aligned}
\left|u_{1}\right|_{\frac{3}{2}+s, \Omega} & \leq C d_{\Omega}^{\frac{1}{2}-s}\left\|\Delta u_{1}\right\|_{0, \Omega} \\
& \leq C d_{\Omega}^{\frac{1}{2}-s}\left(\|f\|_{0, \Omega}+\left(d_{\Omega}^{-2}+k^{2}\right)\|u\|_{0, \Omega}+d_{\Omega}^{-1}|u|_{1, \Omega}\right) \\
& \stackrel{\text { Poincaré }}{\leq} C d_{\Omega}^{\frac{1}{2}-s}\left(\|f\|_{0, \Omega}+k^{2}\|u\|_{0, \Omega}+d_{\Omega}^{-1}|u|_{1, \Omega}\right) \\
& \stackrel{(5)}{\leq} C\left(1+d_{\Omega} k\right)\left(d_{\Omega}^{\frac{1}{2}-s}\|f\|_{0, \Omega}+d_{\Omega}^{-s}\left\|g_{R}\right\|_{0, \Gamma_{R}}\right)
\end{aligned}
$$

where all constants are independent of $k$ and $u$.

Denote by $\widetilde{u}$ and $\widetilde{f}$ the extensions by zero of $u$ and $f$, respectively, to $\Omega_{R}$; clearly, $\widetilde{u} \in H^{1}\left(\Omega_{R}\right)$ and $\widetilde{f} \in L^{2}\left(\Omega_{R}\right)$. The function $u_{2}:=(1-\chi) \widetilde{u}$ solves

$$
\begin{aligned}
-\Delta u_{2}-k^{2} u_{2} & =(1-\chi) \tilde{f}+\widetilde{u} \Delta \chi+2 \nabla \chi \cdot \nabla \widetilde{u} & & \text { in } \Omega_{R} \\
\frac{\partial u_{2}}{\partial \mathbf{n}} \pm i k \vartheta u_{2} & =g_{R} & & \text { on } \Gamma_{R} .
\end{aligned}
$$

The first part of this proof for the case $\Omega_{D}=\emptyset$ applies to $u_{2}$ (denote again by $s_{N}$ the regularity parameter). Thus, since $u_{2}=\tilde{f}=0$ in $\Omega_{D}$ and $u_{2}=u$ on $\Gamma_{R}$, the analogue of estimate (8) implies

$$
\left|u_{2}\right|_{\frac{3}{2}+s, \Omega} \leq C\left(1+d_{\Omega} k\right)\left(d_{\Omega}^{\frac{1}{2}-s}\|f\|_{0, \Omega}+d_{\Omega}^{-s}\left\|g_{R}\right\|_{0, \Gamma_{R}}\right)+C\left\|g_{R}\right\|_{s, \Gamma_{R}} .
$$

Thus, since $u=u_{1}+\left.u_{2}\right|_{\Omega}$, then $u \in H^{\frac{3}{2}+s}(\Omega)$ for all $0<s<s_{\Omega}, s \leq r$, where $s_{\Omega}:=\min \left\{s_{D}, s_{N}\right\}$; moreover, (9) and (10) give the bound (7).

Remark 2.4. Whenever $\Omega_{D}=\emptyset$ and $\Omega_{R}$ is convex, if $r=1 / 2$ then $u \in H^{2}(\Omega)$ and (7) holds with $s=1 / 2($ see $[21,11])$. 


\section{The Trefftz discontinuous Galerkin (TDG) method}

We briefly review the derivation and formulation of our TDG method for the discretization of problem (2), see also [16, Sect. 2] and [14, Sect. 2].

\subsection{Notation}

Let $\mathcal{T}_{h}=\{K\}$ be a finite element partition of $\Omega$, possibly featuring hanging nodes. Its cells are supposed to be affine images of a few simple reference elements, which holds, for instance, for simplicial partitions into triangles $(N=2)$ or tetrahedra $(N=3)$. We write $h$ for the mesh width of $\mathcal{T}_{h}$, i.e, $h=\max _{K \in \mathcal{T}_{h}} h_{K}$, with $h_{K}:=\operatorname{diam}(K)$. On $\mathcal{T}_{h}$ we will define our TDG

method. We denote by $\mathcal{F}_{h}=\bigcup_{K \in \mathcal{T}_{h}} \partial K$ the skeleton of the mesh, and set $\mathcal{F}_{h}^{R}=\mathcal{F}_{h} \cap \Gamma_{R}, \mathcal{F}_{h}^{D}=\mathcal{F}_{h} \cap \Gamma_{D}$ and $\mathcal{F}_{h}^{I}=\mathcal{F}_{h} \backslash\left(\mathcal{F}_{h}^{R} \cup \mathcal{F}_{h}^{D}\right)$.

We also introduce some standard DG notation [1]. Write $\mathbf{n}^{+}, \mathbf{n}^{-}$and $\mathbf{n}_{K}$ for the exterior unit normals on $\partial K^{+}, \partial K^{-}$and $\partial K$, respectively. Let $v$ and $\boldsymbol{\tau}$ be a piecewise smooth function and vector field on $\mathcal{T}_{h}$, respectively. On $\partial K^{-} \cap \partial K^{+}$, we define

the averages: $\left\{\{v\}:=\frac{1}{2}\left(v^{+}+v^{-}\right), \quad\left\{\{\boldsymbol{\tau}\}:=\frac{1}{2}\left(\boldsymbol{\tau}^{+}+\boldsymbol{\tau}^{-}\right)\right.\right.$,

the jumps: $\llbracket v \rrbracket_{N}:=v^{+} \mathbf{n}^{+}+v^{-} \mathbf{n}^{-}, \quad \llbracket \boldsymbol{\tau} \rrbracket_{N}:=\boldsymbol{\tau}^{+} \cdot \mathbf{n}^{+}+\boldsymbol{\tau}^{-} \cdot \mathbf{n}^{-}$.

Furthermore, we will denote by $\nabla_{h}$ the elementwise application of $\nabla$.

Finally, for a given mesh $\mathcal{T}_{h}$, we introduce the TDG finite element spaces with local resolutions $\left\{p_{K}\right\}_{K \in \mathcal{T}_{h}}$ given by

$$
V_{p}\left(\mathcal{T}_{h}\right)=\left\{v_{h p} \in L^{2}(\Omega):\left.v_{h p}\right|_{K} \in V_{p_{K}}(K) \forall K \in \mathcal{T}_{h}\right\}
$$

where $V_{p_{K}}(K)$ is a $p_{K}$-dimensional space of functions on $K$ that enjoy the Trefftz property, i.e.,

$$
-\Delta v_{h p}-k^{2} v_{h p}=0 \quad \forall v_{h p} \in V_{p_{K}}(K) .
$$

Examples of Trefftz spaces for the Helmholtz problems are linear combinations of plane waves in different directions, or linear combinations of circular/spherical waves, see [25, Ch. 3] and Sect. 5.

\subsection{Derivation of the TDG method}

In contrast to $[14,16]$ we derive the TDG method for the scattering problem (2) directly from the second-order equations. Multiplying the first 
equation of (2) by smooth test functions $v$ and integrating by parts on each $K \in \mathcal{T}_{h}$, we obtain

$$
\int_{K}\left(\nabla u \cdot \nabla \bar{v}-k^{2} u \bar{v}\right) \mathrm{d} V-\int_{\partial K} \nabla u \cdot \mathbf{n}_{K} \bar{v} \mathrm{~d} S=0 .
$$

Now, we integrate by parts a second time, replace $u$ and $v$ by discrete functions $u_{h p}, v_{h p} \in V_{p}\left(\mathcal{T}_{h}\right)$ and the traces of $u$ and $\nabla u$ at $\partial K$ by numerical fluxes to be defined $\left(u \rightarrow \widehat{u}_{h p}, \nabla u \rightarrow i k \widehat{\boldsymbol{\sigma}}_{h p}\right)$, and get

$$
\int_{K} u_{h p}\left(-\Delta \bar{v}_{h p}-k^{2} \bar{v}_{h p}\right) \mathrm{d} V+\int_{\partial K} \widehat{u}_{h p} \nabla \bar{v}_{h p} \cdot \mathbf{n}_{K} \mathrm{~d} S-\int_{\partial K} i k \widehat{\boldsymbol{\sigma}}_{h p} \cdot \mathbf{n}_{K} \bar{v}_{h p} \mathrm{~d} S=0 .
$$

Finally, taking into account the Trefftz property (11) of the test functions $v_{h p}$, we can write the elemental TDG formulation:

$$
\int_{\partial K} \widehat{u}_{h p} \nabla \bar{v}_{h p} \cdot \mathbf{n}_{K} \mathrm{~d} S-\int_{\partial K} i k \widehat{\boldsymbol{\sigma}}_{h p} \cdot \mathbf{n}_{K} \bar{v}_{h p} \mathrm{~d} S=0 .
$$

In order to complete the definition of the TDG method, like in [16, Sect. 2], we mimic the general form of the fluxes defined in [6] and set

$$
\begin{aligned}
& i k \widehat{\boldsymbol{\sigma}}_{h p}= \begin{cases}\left.\left\{\nabla_{h} u_{h p}\right\}\right\}-\alpha i k \llbracket u_{h p} \rrbracket_{N} & \text { on faces in } \mathcal{F}_{h}^{I}, \\
\nabla_{h} u_{h p}-(1-\delta)\left(\nabla_{h} u_{h p}+i k \vartheta u_{h p} \mathbf{n}-g_{R} \mathbf{n}\right) & \text { on faces in } \mathcal{F}_{h}^{R}, \\
\nabla_{h} u_{h p}-\alpha i k u_{h p} \mathbf{n} & \text { on faces in } \mathcal{F}_{h}^{D},\end{cases} \\
& \widehat{u}_{h p}= \begin{cases}\left\{\left\{u_{h p}\right\}\right\}-\beta(i k)^{-1} \llbracket \nabla_{h} u_{h p} \rrbracket_{N} & \text { on faces in } \mathcal{F}_{h}^{I}, \\
u_{h p}-\delta\left((i k \vartheta)^{-1} \nabla_{h} u_{h p} \cdot \mathbf{n}+u_{h p}-(i k \vartheta)^{-1} g_{R}\right) & \text { on faces in } \mathcal{F}_{h}^{R}, \\
0 & \text { on faces in } \mathcal{F}_{h}^{D},\end{cases}
\end{aligned}
$$

where the so-called flux parameters $\alpha, \beta, \delta$ are piecewise constant and positive functions defined on suitable unions of edges/faces. In particular, we demand that

$$
0<\delta \leq 1 / 2
$$

More precise assumptions on these flux parameters will be specified later, see (21) below. 
Adding over all elements, we obtain the following formulation of the TDG method: find $u_{h p} \in V_{p}\left(\mathcal{T}_{h}\right)$ such that, for all $v_{h p} \in V_{p}\left(\mathcal{T}_{h}\right)$,

$$
\mathcal{A}_{h}\left(u_{h p}, v_{h p}\right)=\ell_{h}\left(v_{h p}\right),
$$

where

$$
\begin{aligned}
\mathcal{A}_{h}(u, v)= & \int_{\mathcal{F}_{h}^{I}}\left\{\{u\} \llbracket \nabla_{h} \bar{v} \rrbracket_{N} \mathrm{~d} S-\int_{\mathcal{F}_{h}^{I}} \beta(i k)^{-1} \llbracket \nabla_{h} u \rrbracket_{N} \llbracket \nabla_{h} \bar{v} \rrbracket_{N} \mathrm{~d} S\right. \\
& -\int_{\mathcal{F}_{h}^{I}}\left\{\left\{\nabla_{h} u\right\} \cdot \llbracket \bar{v} \rrbracket_{N} \mathrm{~d} S+\int_{\mathcal{F}_{h}^{I}} \alpha i k \llbracket u \rrbracket_{N} \cdot \llbracket \bar{v} \rrbracket_{N} \mathrm{~d} S\right. \\
& +\int_{\mathcal{F}_{h}^{R}}(1-\delta) u \nabla_{h} \bar{v} \cdot \boldsymbol{n} \mathrm{d} S-\int_{\mathcal{F}_{h}^{R}} \delta(i k \vartheta)^{-1}\left(\nabla_{h} u \cdot \boldsymbol{n}\right)\left(\nabla_{h} \bar{v} \cdot \boldsymbol{n}\right) \mathrm{d} S \\
& -\int_{\mathcal{F}_{h}^{R}} \delta \nabla_{h} u \cdot \boldsymbol{n} \bar{v} \mathrm{~d} S+\int_{\mathcal{F}_{h}^{R}}(1-\delta) i k \vartheta u \bar{v} \mathrm{~d} S \\
& -\int_{\mathcal{F}_{h}^{D}} \nabla_{h} u \cdot \boldsymbol{n} \bar{v} \mathrm{~d} S+\int_{\mathcal{F}_{h}^{D}} \alpha i k u \bar{v} \mathrm{~d} S,
\end{aligned}
$$

and

$$
\ell_{h}(v)=-\int_{\mathcal{F}_{h}^{R}} \delta(i k \vartheta)^{-1} g_{R} \nabla_{h} \bar{v} \cdot \boldsymbol{n} \mathrm{d} S+\int_{\mathcal{F}_{h}^{R}}(1-\delta) g_{R} \bar{v} \mathrm{~d} S .
$$

Remark 3.1. We have local approximation by plane waves in mind, which is unstable in the limit $k \rightarrow 0$. Thus, throughout, we take for granted that $k$ is bounded away from zero and forgo a definition of our method that would be robust in the limit $k \rightarrow 0$. Yet, this can easily be achieved by replacing $i k$ with $i k+h^{-1}$ in the above definitions of the numerical fluxes, which yields a viable DG formulation also for $k=0$.

\section{A priori convergence analysis}

Well-posedness and abstract a priori error estimates for the TDG method introduced in the previous section are proved essentially like in [16] (see also [25, Sec. 4.3]), with judicious modifications due to the presence of the Dirichlet boundary $\Gamma_{D}$ and the reduced regularity of the analytical solutions.

Define the piecewise Trefftz space

$$
T\left(\mathcal{T}_{h}\right):=\left\{v \in L^{2}(\Omega): \exists s>0 \text { s.t. } v \in H^{\frac{3}{2}+s}\left(\mathcal{T}_{h}\right)\right.
$$


and $\Delta v+k^{2} v=0$ in each $\left.K \in \mathcal{T}_{h}\right\}$,

where $H^{s}\left(\mathcal{T}_{h}\right)=\left\{v \in L^{2}(\Omega):\left.v\right|_{K} \in H^{s}(K) \forall K \in \mathcal{T}_{h}\right\}$, and endow it with the mesh-skeleton norm, see [16, Formula (3.1)],

$$
\begin{aligned}
& \|v\|_{D G}^{2}:=k^{-1}\left\|\beta^{\frac{1}{2}} \llbracket \nabla_{h} v \rrbracket_{N}\right\|_{0, \mathcal{F}_{h}^{I}}^{2}+k\left\|\alpha^{\frac{1}{2}} \llbracket v \rrbracket_{N}\right\|_{0, \mathcal{F}_{h}^{I}}^{2} \\
& +k^{-1}\left\|\delta^{\frac{1}{2}} \vartheta^{-\frac{1}{2}} \nabla_{h} v \cdot \mathbf{n}\right\|_{0, \mathcal{F}_{h}^{R}}^{2}+k\left\|(1-\delta)^{\frac{1}{2}} \vartheta^{\frac{1}{2}} v\right\|_{0, \mathcal{F}_{h}^{R}}^{2} \\
& +k\left\|\alpha^{\frac{1}{2}} v\right\|_{0, \mathcal{F}_{h}^{D}}^{2}
\end{aligned}
$$

this is actually a norm in $T\left(\mathcal{T}_{h}\right)$. In fact, if $v \in T\left(\mathcal{T}_{h}\right)$ is such that $\|v\|_{D G}=0$, then $v \in H_{0}^{1}(\Omega), \nabla v \in H(\operatorname{div} ; \Omega), \nabla v \cdot \mathbf{n}=0$ on $\Gamma_{R}$ and $\Delta v+k^{2} v=0$, thus $v=0$ as a consequence of the well-posedness of problem (2).

In order to study the properties of $\mathcal{A}_{h}(\cdot, \cdot)$, we also need to introduce the augmented $D G$-norm, cf. [16, Formula (3.2)],

$$
\begin{aligned}
\|v\|_{D G^{+}}^{2}= & \|v\|_{D G}^{2}+k \| \beta^{-\frac{1}{2}}\left\{\{v\}\left\|_{0, \mathcal{F}_{h}^{I}}^{2}+k^{-1}\right\| \alpha^{-\frac{1}{2}}\left\{\left\{\nabla_{h} v\right\}\right\} \|_{0, \mathcal{F}_{h}^{I}}^{2}\right. \\
& +k\left\|\delta^{-\frac{1}{2}} \vartheta^{\frac{1}{2}} v\right\|_{0, \mathcal{F}_{h}^{R}}^{2}+k^{-1}\left\|\alpha^{-\frac{1}{2}} \nabla_{h} v \cdot \mathbf{n}\right\|_{0, \mathcal{F}_{h}^{D}}^{2} .
\end{aligned}
$$

Proposition 4.1. For all $v, w \in T\left(\mathcal{T}_{h}\right)$ we have

$$
\begin{aligned}
\left|\mathcal{A}_{h}(v, w)\right| & \leq 2\|v\|_{D G^{+}}\|w\|_{D G}, \\
\operatorname{Im}\left[\mathcal{A}_{h}(v, v)\right] & =\|v\|_{D G}^{2} .
\end{aligned}
$$

Proof. Repeated application of the Cauchy-Schwarz inequality and $\delta \leq(1-$ $\delta)<1$ give (16) (see [16, Prop. 3.5] and [25, Prop. 4.3.5]), while (17) can be proved by integrating by parts the volume term in (12) and proceeding like in [16, Prop. 3.3] (see also [25, Prop. 4.3.3]).

\subsection{Well-posedness and error estimates in DG-norm}

In this section, we state the well-posedness of the TDG method and an abstract error estimate in the $D G$-norm (15).

Proposition 4.2. There exists a unique $u_{h p}$ solution to $(14)$; moreover, we have

$$
\left\|u_{h p}\right\|_{D G} \leq\left\|(1-\delta)^{\frac{1}{2}} \vartheta^{-\frac{1}{2}} g_{R}\right\|_{0, \mathcal{F}_{h}^{R}} .
$$


Proof. Existence and uniqueness of solutions to (14) readily follows from (17). By the Cauchy-Schwarz inequality and $\delta \leq(1-\delta)$, see $(13)$, we obtain

$$
\left|\ell_{h}(v)\right| \leq\left\|(1-\delta)^{\frac{1}{2}} \vartheta^{-\frac{1}{2}} g_{R}\right\|_{0, \mathcal{F}_{h}^{R}}\|v\|_{D G}
$$

which, together with (17), gives the continuous dependence of $u_{h p}$ on $g_{R}$.

Proposition 4.3. Assume that the solution $u$ of (2) belongs to $T\left(\mathcal{T}_{h}\right)$ and let $u_{h p}$ be the solution to (14); then, the TDG formulation is consistent, i.e.,

$$
\mathcal{A}_{h}\left(u, v_{h p}\right)=\ell_{h}\left(v_{h p}\right) \quad \forall v_{h p} \in V_{p}\left(\mathcal{T}_{h}\right)
$$

and

$$
\left\|u-u_{h p}\right\|_{D G} \leq 3 \inf _{v_{h p} \in V_{p}\left(\mathcal{T}_{h}\right)}\left\|u-v_{h p}\right\|_{D G^{+}} .
$$

Proof. The consistency of the TDG formulation is a consequence of the consistency of the numerical fluxes. For the proof of the error estimate, see [16, Prop. 3.6] and [25, Prop. 4.3.6].

\subsection{Error estimates in the $L^{2}$-norm}

Like in [16, Sect. 3.2] (see also [25, Sec. 4.3]), we bound the $L^{2}$-norm of any Trefftz function by using a modified duality argument which was introduced in [29, Theorem 3.1] for convex polygonal $(N=2)$ or polyhedral $(N=3)$ domains, and extended to non convex polygons in [12, Lemma 6.2 (ii)]. Since we want to allow for meshes which can be highly refined close to the scatterer, we dispense with the quasi-uniformity assumption. This will entail major modifications in the arguments compared to the analysis of [16] and [25, Sec. 4.3].

We make the following assumptions on the mesh:

i) shape regularity: there exists a constant $\sigma \geq 1$ independent of the global mesh width $h$ such that

$$
\max _{K \in \mathcal{T}_{h}} \frac{h_{K}}{d_{K}} \leq \sigma
$$

where $d_{K}$ is the diameter of the largest ball contained in $K$;

ii) local quasi-uniformity: there exists a constant $\tau \geq 1$ independent of $h$ such that

$$
\tau^{-1} \leq \frac{h_{K_{1}}}{h_{K_{2}}} \leq \tau \quad \forall K_{1}, K_{2} \in \mathcal{T}_{h} \text { s.t. } \operatorname{meas}_{(N-1)}\left(\partial K_{1} \cap \partial K_{2}\right) \neq 0 ;
$$


iii) quasi-uniformity close to $\Gamma_{R}$ : there exists a constant $\tau_{R} \geq 1$ independent of $h$ such that

$$
\frac{h}{h_{K}} \leq \tau_{R} \quad \forall K \in \mathcal{T}_{h} \text { s.t. } \operatorname{meas}_{(N-1)}\left(\partial K \cap \Gamma_{R}\right) \neq 0
$$

Note that most local refinement algorithms for finite element meshes in two and three dimensions are designed to ensure Assumptions i) and ii). Assumption iii) stipulates that all the elements abutting the outer boundary $\Gamma_{R}$ have size comparable to $h$. Yet, what is important in applications is the possibility of refining the meshes close to the scatterer, rather than close to the artificially introduced outer boundary, which will often be smooth. Figure 1 displays a mesh compliant with i)-iii).

In [16, Sect. 5] the numerical flux parameters $\alpha, \beta, \delta \in L^{\infty}\left(\mathcal{F}_{h}\right)$ were chosen as globally constant functions. To cope with locally refined meshes we now allow them to attain different values for different edges $(N=2)$ or faces $(N=3)$ and we assume that those have the following form $(f$ is an edge/face of the mesh):

$$
\left.\alpha\right|_{f}=\mathrm{a} \frac{h}{h_{f}},\left.\quad \beta\right|_{f}=\mathrm{b} \frac{h}{h_{f}},\left.\quad \delta\right|_{f}=\mathrm{d} \frac{h}{h_{f}},
$$

where $\mathrm{a}, \mathrm{b}$ and $\mathrm{d}$ are positive constants independent of the mesh width, the local Trefftz trial spaces, and the wave number $k$. The symbol $h_{f}$ stands for the local mesh width at the edge/face $f$ defined as $h_{f}=\min \left\{h_{K_{1}}, h_{K_{2}}\right\}$ if $f=\partial K_{1} \cap \partial K_{2}$, and $h_{f}=h_{K}$ if $f=\partial K \cap \partial \Omega$. Notice that this definition works also in the case of hanging nodes.

Notice that the condition $\delta \leq 1 / 2$ is satisfied by choosing $\mathrm{d} \leq\left(2 \tau_{R}\right)^{-1}$ with $\tau_{R}$ from (20). This is the rationale behind assuming (20).

Lemma 4.4. There exists a constant $C>0$ depending only on $\sigma, s, \vartheta, \gamma_{R}$ and the flux parameters $\mathrm{a}, \mathrm{b}$ and $\mathrm{d}$ (thus independent of $h, p$ and $k$ ), such that, for any $w \in T\left(\mathcal{T}_{h}\right)$,

$$
\|w\|_{0, \Omega} \leq C \tau^{\frac{1}{2}} d_{\Omega}\left[(k h)^{-\frac{1}{2}}+\left(d_{\Omega} k\right)^{\frac{1}{2}}\left(d_{\Omega}^{-1} h\right)^{s}\right]\|w\|_{D G}
$$

for all s satisfying (6).

Proof. Let $\phi$ be in $L^{2}(\Omega)$. Let $v$ be the solution to the (adjoint) problem (3) with $f=\phi, g_{R}=0$ and "-" in the impedance condition on $\Gamma_{R}$. From (5) 
and Theorem 2.3, we know that $v \in H^{\frac{3}{2}+s}(\Omega)$, for all $s$ satisfying (6) (with $\left.\min \left\{s_{\Omega}, r\right\}=s_{\Omega}\right)$, and that

$$
\begin{aligned}
|v|_{1, \Omega}+k\|v\|_{0, \Omega} & \leq C d_{\Omega}\|\phi\|_{0, \Omega}, \\
|\nabla v|_{\frac{1}{2}+s, \Omega} & \leq C\left(1+d_{\Omega} k\right) d_{\Omega}^{\frac{1}{2}-s}\|\phi\|_{0, \Omega},
\end{aligned}
$$

with $C>0$ depending only on $s, \gamma_{R}$ and $\vartheta$, but independent of $k, \phi$ and $v$. Multiplying by $w \in T\left(\mathcal{T}_{h}\right)$, integrating by parts twice the first equation of (3) element by element (using $\Delta w+k^{2} w=0$ in each $K \in \mathcal{T}_{h}$ ), and taking into account that $\nabla v \cdot \boldsymbol{n}=i k \vartheta v$ on $\Gamma_{R}$ and $v=0$ on $\Gamma_{D}$, we obtain

$$
\begin{aligned}
\left|(w, \phi)_{0, \Omega}\right|= & \left|\sum_{K \in \mathcal{T}_{h}} \int_{\partial K}(\nabla w \cdot \boldsymbol{n} \bar{v}-w \overline{\nabla v \cdot \boldsymbol{n}}) \mathrm{d} S\right| \\
= & \mid \int_{\mathcal{F}_{h}^{I}}\left(\llbracket \nabla_{h} w \rrbracket_{N} \bar{v}-\llbracket w \rrbracket_{N} \cdot \overline{\nabla v}\right) \mathrm{d} S \\
& +\int_{\mathcal{F}_{h}^{R}}\left(\nabla_{h} w \cdot \boldsymbol{n}+i k \vartheta w\right) \bar{v} \mathrm{~d} S-\int_{\mathcal{F}_{h}^{D}} w \overline{\nabla v} \cdot \mathbf{n} \mathrm{d} S \mid,
\end{aligned}
$$

from which, by the Cauchy-Schwarz inequality,

$$
\begin{aligned}
& \left|(w, \phi)_{0, \Omega}\right| \leq \sum_{f \in \mathcal{F}_{h}^{I}}\left(k^{-\frac{1}{2}}\left\|\beta^{\frac{1}{2}} \llbracket \nabla_{h} w \rrbracket_{N}\right\|_{0, f} k^{\frac{1}{2}}\left\|\beta^{-\frac{1}{2}} v\right\|_{0, f}\right.
\end{aligned}
$$

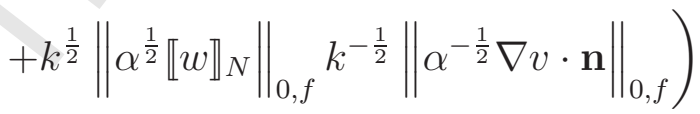

$$
\begin{aligned}
& +\sum_{f \in \mathcal{F}_{h}^{R}}\left(k^{-\frac{1}{2}}\left\|\delta^{\frac{1}{2}} \vartheta^{-\frac{1}{2}} \nabla w \cdot \boldsymbol{n}\right\|_{0, f} k^{\frac{1}{2}}\left\|\delta^{-\frac{1}{2}} \vartheta^{\frac{1}{2}} v\right\|_{0, f}\right. \\
& \left.+k^{\frac{1}{2}}\left\|\delta^{\frac{1}{2}} \vartheta^{\frac{1}{2}} w\right\|_{0, f} k^{\frac{1}{2}}\left\|\delta^{-\frac{1}{2}} \vartheta^{\frac{1}{2}} v\right\|_{0, f}\right) \\
& +\sum_{f \in \mathcal{F}_{h}^{D}} k^{\frac{1}{2}}\left\|\alpha^{\frac{1}{2}} w\right\|_{0, f} k^{-\frac{1}{2}}\left\|\alpha^{-\frac{1}{2}} \nabla v \cdot \mathbf{n}\right\|_{0, f} \\
& \leq\|w\|_{D G} \mathcal{G}(v)^{\frac{1}{2}},
\end{aligned}
$$

where we have set

$$
\mathcal{G}(v):=\sum_{f \in \mathcal{F}_{h}^{I}}\left(k\left\|\beta^{-\frac{1}{2}} v\right\|_{0, f}^{2}+k^{-1}\left\|\alpha^{-\frac{1}{2}} \nabla v \cdot \mathbf{n}\right\|_{0, f}^{2}\right)
$$




$$
+\sum_{f \in \mathcal{F}_{h}^{R}} 2 k\left\|\delta^{-\frac{1}{2}} \vartheta^{\frac{1}{2}} v\right\|_{0, f}^{2}+\sum_{f \in \mathcal{F}_{h}^{D}} k^{-1}\left\|\alpha^{-\frac{1}{2}} \nabla v \cdot \mathbf{n}\right\|_{0, f}^{2} .
$$

We recall the trace inequality

$$
\|v\|_{0, \partial K}^{2} \leq C\left(h_{K}^{-1}\|v\|_{0, K}^{2}+h_{K}|v|_{1, K}^{2}\right) \quad \forall K \in \mathcal{T}_{h}
$$

with $C>0$ depending only on $\sigma$ in (18) (see [4, Th. 1.6.6]). Moreover, since $v \in H^{\frac{3}{2}+s}(\Omega)$ for all $s$ satisfying $(6)$, we can prove that

$$
\|\nabla v\|_{0, \partial K}^{2} \leq C\left(h_{K}^{-1}\|\nabla v\|_{0, K}^{2}+h_{K}^{2 s}|\nabla v|_{\frac{1}{2}+s, K}^{2}\right) \quad \forall K \in \mathcal{T}_{h},
$$

with $C>0$ depending only on $\sigma, s$, and the reference element associated with $K$. In fact, denoting by $\widehat{K}$ the reference element associated with $K$ (recall that any $K$ is the affine image of one of a few reference elements), from $\left[22\right.$, Th. A.2], we have that, for any $\widehat{w} \in H^{\frac{1}{2}+s}(\widehat{K})$,

$$
\|\widehat{w}\|_{0, \partial \widehat{K}}^{2} \leq C\|\widehat{w}\|_{0, \widehat{K}}^{4 s /(1+2 s)}\|\widehat{w}\|_{\frac{1}{2}+s, \widehat{K}}^{2 /(1+2 s)}
$$

Bounding the $L^{2}$-norm by the $H^{\frac{1}{2}+s}(\widehat{K})$-norm gives $\|\widehat{w}\|_{0, \partial \widehat{K}}^{2} \leq C\|\widehat{w}\|_{\frac{1}{2}+s, \widehat{K}}^{2}$ which, together with the definition of the $H^{\frac{1}{2}+s}(\widehat{K})$-norm, gives $\|\widehat{w}\|_{0, \partial \widehat{K}}^{2} \leq$ $C\left(\|\widehat{w}\|_{0, \widehat{K}}^{2}+|\widehat{w}|_{\frac{1}{2}+s, \widehat{K}}^{2}\right)$. By a scaling argument, which is possible thanks to the shape-regularity (18), taking into account that $\nabla v \in H^{\frac{1}{2}+s}(\Omega)$, we obtain (24).

Using (23) and (24), and taking into account the bounds for $\vartheta$ and the local quasi-uniformity assumption (19), we obtain

$$
\begin{aligned}
\mathcal{G}(v) \leq C \tau \sum_{K \in \mathcal{T}_{h}}( & \left\|\alpha^{-\frac{1}{2}}\right\|_{L^{\infty}\left(\partial K \cap\left(\mathcal{F}_{h}^{I} \cup \mathcal{F}_{h}^{D}\right)\right)}^{2}+\left\|\beta^{-\frac{1}{2}}\right\|_{L^{\infty}\left(\partial K \cap \mathcal{F}_{h}^{I}\right)}^{2} \\
+ & \left.\left\|\delta^{-\frac{1}{2}}\right\|_{L^{\infty}\left(\partial K \cap \mathcal{F}_{h}^{R}\right)}^{2}\right) \\
& \cdot\left[\frac{k}{h_{K}}\|v\|_{0, K}^{2}+\left(k h_{K}+\frac{1}{k h_{K}}\right)|v|_{1, K}^{2}+\frac{h_{K}^{2 s}}{k}|\nabla v|_{\frac{1}{2}+s, K}^{2}\right],
\end{aligned}
$$

with $C>0$ depending only on $\vartheta, s$ and $\sigma$ (see (18)), but independent of $h$, $p, k$ and $v$. The assumption (21) on the flux parameters immediately gives

$$
\left\|\alpha^{-\frac{1}{2}}\right\|_{L^{\infty}\left(\partial K \cap\left(\mathcal{F}_{h}^{I} \cup \mathcal{F}_{h}^{D}\right)\right)}^{2},\left\|\beta^{-\frac{1}{2}}\right\|_{L^{\infty}\left(\partial K \cap \mathcal{F}_{h}^{I}\right)}^{2},\left\|\delta^{-\frac{1}{2}}\right\|_{L^{\infty}\left(\partial K \cap \mathcal{F}_{h}^{R}\right)}^{2} \leq C \frac{h_{K}}{h},
$$


which leads to the estimate

$$
\mathcal{G}(v) \leq C \tau \sum_{K \in \mathcal{T}_{h}}\left[\frac{k}{h}\|v\|_{0, K}^{2}+\left(\frac{k h_{K}^{2}}{h}+\frac{1}{k h}\right)|v|_{1, K}^{2}+\frac{h_{K}^{2 s+1}}{k h}|\nabla v|_{\frac{1}{2}+s, K}^{2}\right],
$$

where $C$ now also depends on a, b and d. By definition, $h_{K} \leq h$, and therefore (22) gives

$$
\mathcal{G}(v) \leq C \tau d_{\Omega}^{2}\left(k^{-1} h^{-1}+d_{\Omega}^{1-2 s} k h^{2 s}\right)\|\phi\|_{0, \Omega}^{2} .
$$

Consequently, for all $\phi \in L^{2}(\Omega)$, we obtain

$$
\frac{\left|(w, \phi)_{0, \Omega}\right|}{\|\phi\|_{0, \Omega}} \leq C \tau^{\frac{1}{2}} d_{\Omega}\left(k^{-\frac{1}{2}} h^{-\frac{1}{2}}+d_{\Omega}^{\frac{1}{2}-s} k^{\frac{1}{2}} h^{s}\right)\|w\|_{D G}
$$

and the result readily follows.

Theorem 4.5. Assume that the analytical solution $u$ to $(2)$ belongs to $T\left(\mathcal{T}_{h}\right)$ and let $u_{h p}$ be the solution to (14); then, there exists a constant $C>0$ depending only on $\sigma, s, \vartheta, \gamma_{R}$ and the flux parameters $\mathrm{a}, \mathrm{b}$ and $\mathrm{d}$ (thus independent of $h, p$ and $k)$, such that

$$
\left\|u-u_{h p}\right\|_{0, \Omega} \leq C \tau^{\frac{1}{2}} d_{\Omega}\left[(k h)^{-\frac{1}{2}}+\left(d_{\Omega} k\right)^{\frac{1}{2}}\left(d_{\Omega}^{-1} h\right)^{s}\right] \inf _{v_{h p} \in V_{p}\left(\mathcal{T}_{h}\right)}\left\|u-v_{h p}\right\|_{D G^{+}}
$$

for all s satisfying (6).

Proof. The result follows from Lemma 4.4 applied to $u-u_{h p} \in T\left(\mathcal{T}_{h}\right)$ and Proposition 4.3.

Remark 4.6. In order to model the propagation of a wave through different materials, it is possible to consider a piecewise constant wavenumber $k$ in the boundary value problem (2). If the mesh $\mathcal{T}_{h}$ resolves the material structure, i.e., in the interior of every element the wavenumber is constant, the formulation of the TDG method and the most part of its analysis carried out in this paper can be extended to this setting after small modification (e.g., $k$ must be placed inside the jump and the average operators in the definition of the numerical fluxes). On the other hand, Rellich identities are not available, at the moment, for variable $k$. Therefore we can not state a variable wavenumber-explicit stability bound analogous to Theorem 2.2. In summary, all the results of this paper carry over to this setting, but the dependence 
on the wavenumber of the bounding constants is not known in the following parts: stability and regularity Theorems 2.2 and 2.3; duality argument of Lemma $4.4 ; L^{2}(\Omega)$-norm error estimate of Theorem $4.5 ; L^{2}(\Omega)$-orders of convergence in the bound (29).

Remark 4.7. In principle, it is possible to apply the TDG scheme to the inhomogeneous Helmholtz equation, i.e., $-\Delta u-k^{2} u=f$ with a non-zero volume source term $f \in L^{2}(\Omega)$. In [14], convergence in $h$ for the plane wavebased TDG was proved but the numerical results presented there clearly demonstrate that no $p$-convergence or high-order $h$-convergence (using many plane waves per element) are possible (see also Remark 13 and Table 3.4 of [7] for the same results for the UWVF). Since the solution of the inhomogeneous problem does not lie in the Trefftz space, the same negative result must be expected for any Trefftz scheme. Other approaches are possible. In [32, Sec. 6.1.2] and subsequent papers, the original problem was reduced to a homogeneous one by subtracting a free-space solution (i.e. a solution of the $\mathrm{PDE}$ in $\mathbb{R}^{N}$ without boundary conditions). Another option is to include non-Trefftz functions (e.g. polynomials) in the discrete space; some volume terms must be included in the formulation of the TDG in order to maintain consistency. However, the analysis of this scheme is still open.

\section{Outlook: $h p$-approximation estimates}

As mentioned in the introduction, the results presented in the previous sections are essential tools for a complete $h p$-convergence theory of the TDG method for the acoustic scattering problem. In fact, with Theorem 4.5 on hand, predicting the convergence of a TDG discretization on $h p$-meshes and distribution of the local resolutions boils down to investigation of the best approximation error $\inf _{v_{h p} \in V_{p}\left(\mathcal{T}_{h}\right)}\left\|u-v_{h p}\right\|_{D G^{+}}$for a concrete choice of local Trefftz spaces.

In this section, we consider spaces spanned by plane waves and review the best approximation results of [26, 27] (see also [25, Ch. 3]) for solutions which are at least in $H^{2}(\Omega)$. What is still missing are $h$-version plane wave local best approximation estimates for Helmholtz solutions in $H^{\frac{3}{2}+s}, 0<s<1 / 2$, to be used within elements abutting corners of $\Gamma_{D}$, where the solution $u$ has low regularity.

In order to avoid technicalities, we restrict ourselves to two-dimensional domains, meshes whose elements are affine images of a few convex reference 
elements, and to uniformly spaced plane wave propagation directions ${ }^{5}$.

For each element $K \in \mathcal{T}_{h}$, define a local "plane wave degree" $q_{K} \in \mathbb{N}$ and a Trefftz space with $p_{K}:=2 q_{K}+1$ uniformly spaced plane waves:

$V_{p}\left(\mathcal{T}_{h}\right):=\left\{v_{h p} \in L^{2}(\Omega):\left.v_{h p}\right|_{K}(\mathbf{x})=\sum_{n=1}^{p_{K}} \alpha_{K, n} e^{i k \mathbf{x} \cdot \mathbf{d}_{K, n}}, \alpha_{K, n} \in \mathbb{C} \forall K \in \mathcal{T}_{h}\right\}$,

where

$$
\mathbf{d}_{K, n}=\left(\cos \frac{2 \pi n}{p_{K}}, \sin \frac{2 \pi n}{p_{K}}\right), \quad n \in\left\{1, \ldots, p_{K}\right\} .
$$

Assume that, in each element,

$$
\left.u\right|_{K} \in H^{s_{K}+1}(K), \quad 1 \leq s_{K} \in \mathbb{N} .
$$

Assume also that

$$
q_{K} \geq 2 s_{K}+1 \quad \forall K \in \mathcal{T}_{h},
$$

and set

$$
\widehat{q}_{K}:=\frac{q_{K}}{\log \left(q_{K}+2\right)} .
$$

With the new definition of the flux parameters $\alpha, \beta$ and $\delta$ (see (21)), and for $h_{K}$ and $q_{K}$ varying across the mesh, the best approximation estimate in $D G^{+}$-norm of [25, Lemma 4.4.1] becomes

$$
\begin{aligned}
\inf _{v_{h p} \in V_{p}\left(\mathcal{T}_{h}\right)} & \left\|u-v_{h p}\right\|_{D G^{+}} \leq \tau^{\frac{1}{2}} k^{-\frac{1}{2}} \sum_{K \in \mathcal{T}_{h}} \widetilde{C}_{K}\left(1+\left(k h_{K}\right)^{\frac{15}{2}}\right) e^{\frac{7}{4} k h_{K}}\left(\frac{h}{h_{K}}\right)^{\frac{1}{2}} h_{K}^{s_{K}-\frac{1}{2}} \\
\cdot & {\left[\left(\frac{1}{\widehat{q}_{K}}\right)^{s_{K}-\frac{1}{2}}+\left(1+\left(k h_{K}\right)^{q_{K}-s_{K}+2}\right)\left(\frac{\sigma e}{2\left(q_{K}+1\right)}\right)^{\frac{q_{K}}{2}}\right]\|u\|_{s_{K}+1, k, K} }
\end{aligned}
$$

where, for every $K \in \mathcal{T}_{h}$, the constants $\widetilde{C}_{K}>0$ depend only on the reference element for $K, s_{K}$ and the maximum and minimum values on $\partial K$ of a, b, d and $\vartheta$.

\footnotetext{
${ }^{5}$ Everything that follows can be generalized to three dimensional problems, meshes with star-shaped elements, general choices of plane wave propagation directions, and also to circular and spherical wave bases.
} 
The first term in the square brackets of (27) decays algebraically for increasing $q_{K}$, while the second one decays exponentially; thus, for $q_{K}$ large enough, the latter can be bounded by the former. This is the situation we consider in the following proposition, which is obtained by inserting the bound (27) within Proposition 4.3 and Theorem 4.5.

Proposition 5.1. Assume that the analytical solution $u$ to (2) satisfies the smoothness assumption (26), and let $u_{h p}$ be the solution to (14) with $V_{p}\left(\mathcal{T}_{h}\right)$ given by (25). Then, if $\min _{K \in \mathcal{T}_{h}} q_{K}$ is large enough (i.e., such that the exponential term in $q_{K}$ in the square bracket of (27) is bounded by the algebraic one), the following error bounds hold true:

$$
\begin{gathered}
\left\|u-u_{h p}\right\|_{D G^{+}} \leq\left(\frac{\tau h}{k}\right)^{\frac{1}{2}} \sum_{K \in \mathcal{T}_{h}} C_{K} h_{K}^{s_{K}-1}\left(\frac{1}{\widehat{q}_{K}}\right)^{s_{K}-\frac{1}{2}}\|u\|_{s_{K}+1, k, K} \\
\left\|u-u_{h p}\right\|_{0, \Omega} \leq C_{*} \tau d_{\Omega}^{2}\left[\left(d_{\Omega} k\right)^{-1}+\left(d_{\Omega}^{-1} h\right)^{s+\frac{1}{2}}\right] \\
\cdot \sum_{K \in \mathcal{T}_{h}} C_{K} h_{K}^{s_{K}-1}\left(\frac{1}{\widehat{q}_{K}}\right)^{s_{K}-\frac{1}{2}}\|u\|_{s_{K}+1, k, K}
\end{gathered}
$$

for all $s$ satisfying (6). Here, for every $K \in \mathcal{T}_{h}$, the local constants $C_{K}>0$ depend only on the product $k h_{K}$ (as increasing functions), $s_{K}$, the reference element for $K$, and the maximum and minimum values on $\partial K$ of $\mathrm{a}, \mathrm{b}, \mathrm{d}$ and $\vartheta$; the global constant $C_{*}>0$ depends only on $\sigma, s, \vartheta, \gamma_{R}$ and the flux parameters $\mathrm{a}, \mathrm{b}$ and $\mathrm{d}$.

The plane wave local best approximation error estimates used to prove the bound (27) provide algebraic orders of convergence in $q_{K}$ when the solution is $H^{s_{K}+1}(K)$ in $K$. Whenever $\left.u\right|_{K}$ admits an analytic extension to an open set $E_{K} \supset \bar{K}$, then the convergence there is exponential in $q_{K}$, as shown in [25, Remark 3.5.8]. Thus, defining

$$
\begin{aligned}
& \mathcal{T}_{h}^{E}:=\left\{K \in \mathcal{T}_{h}:\left.u\right|_{K} \text { can be analytically extended to } E_{K}\right\}, \\
& \mathcal{T}_{h}^{A}:=\mathcal{T}_{h} \backslash \mathcal{T}_{h}^{E},
\end{aligned}
$$

the combination of [25, Remark 3.5.8] with Proposition 4.3 gives the following error bound, whose convergence in $q_{K}$ is faster than that of (28) in the part of the domain occupied by the elements in $\mathcal{T}_{h}^{E}$ :

$\left\|u-u_{h p}\right\|_{D G^{+}} \leq\left(\frac{\tau h}{k}\right)^{\frac{1}{2}}\left[\sum_{K \in \mathcal{T}_{h}^{A}} C_{K}^{A} h_{K}^{s_{K}-1}\left(\frac{1}{\widehat{q}_{K}}\right)^{s_{K}-\frac{1}{2}}\|u\|_{s_{K}+1, k, K}\right.$ 


$$
\left.+\sum_{K \in \mathcal{T}_{h}^{E}} C_{K}^{E}\left(1+\left(k h_{K}\right)^{q_{K}}\right) b_{K}^{-q_{K}}\left(\|u\|_{s_{K}+1, k, K}+\left\|u_{K}^{*}\right\|_{L^{\infty}\left(E_{K}\right)}\right)\right]
$$

where $u_{K}^{*}$ is the extension of $\left.u\right|_{K}$ to $E_{K}$. Here, the local constants $C_{K}^{A}, C_{K}^{E}>0$ are independent of $q_{K}$ and $u$, and depend only on the product $k h_{K}$ (as increasing functions), the reference element for $K, s_{K},\left.\mathrm{a}\right|_{\partial K},\left.\mathrm{~b}\right|_{\partial K},\left.\mathrm{~d}\right|_{\partial K}$, $\left.\vartheta\right|_{\partial K}$ and, in the case of $C_{K}^{E}$, on $h_{K}$ and $E_{K} ; b_{K}>1$ depends only on $K$ and $E_{K}$. A similar bound on the $L^{2}(\Omega)$-norm of the error follows from Theorem 4.5. If the artificial boundary $\Gamma_{R}$ is chosen to be analytic, we expect $\mathcal{T}_{h}^{A}$ to correspond to the set of the elements of $\mathcal{T}_{h}$ that are incident to a vertex of $\Gamma_{D}$.

We hope that the above estimates, together with local best approximation estimates for $H^{\frac{3}{2}+s}$-Helmholtz solutions, $0<s<1 / 2$, may serve as tools for a proof of exponential convergence of plane wave TDG on $h p$-meshes and suitable plane wave degree distributions. Such a result would match what has been established for piecewise polynomial approximation $[2,35]$. Hitherto it is still elusive.

Remark 5.2. In order to obtain an accurate approximation of the solution near the vertices of a polygonal scatterer, a possible alternative to the use of locally refined meshes is the use of Trefftz trial functions specially adapted to the geometry. These are the so-called "corner waves" and they are defined as product of Bessel function of fractional order in the radial direction and complex exponentials in the angular direction. They exhibit a singularity located in the corner, with the same exponent that is expected for the analytical solution of the boundary value problem.

This approach is very simple and provides extremely effective results, as demonstrated in [3]. However, the extension to three dimensional problems is absolutely nontrivial: this fact gives a further motivation to the study of a $h p$-version of the TDG method.

\section{Conclusions}

In the present paper, we have considered a sound soft scattering problem posed in a truncated domain and proved wavenumber-explicit stability and regularity bounds in $H^{\frac{3}{2}+s}$-norm, $s>0$. We have derived a TDG discretization and proved its well-posedness and quasi-optimality in a special DG norm. Quasi-optimality in $L^{2}$-norm was then proved for shape regular, locally quasi-uniform (but not globally quasi-uniform) meshes. We have 
shown convergence estimates in $h$ and $p$ in a special case which uses plane wave bases.

Several aspects still remain elusive, for example: the proof of exponential convergence in the number of degrees of freedom on special meshes, and the extension to inhomogeneous problems and non homogeneous materials.

[1] D.N. Arnold, F. Brezzi, B. Cockburn, L.D. Marini, Unified analysis of discontinuous Galerkin methods for elliptic problems, SIAM J. Numer. Anal. 39 (2002) 1749-1779.

[2] I. Babuška, M. Suri, The $p$ and $h-p$ versions of the finite element method, basic principles and properties, SIAM Rev. 36 (1994) 578-632.

[3] A.H. Barnett, T. Betcke, An exponentially convergent nonpolynomial finite element method for time-harmonic scattering from polygons, SIAM J. Sci. Comput. 32 (2010) 1417-1441.

[4] S.C. Brenner, L.R. Scott, Mathematical theory of finite element methods, 3rd ed., Texts Appl. Math., Springer-Verlag, New York, 2007.

[5] A. Buffa, P. Monk, Error estimates for the ultra weak variational formulation of the Helmholtz equation, M2AN, Math. Model. Numer. Anal. 42 (2008) 925-940.

[6] P. Castillo, B. Cockburn, I. Perugia, D. Schötzau, An a priori error analysis of the local discontinuous Galerkin method for elliptic problems, SIAM J. Numer. Anal. 38 (2000) 1676-1706.

[7] O. Cessenat, B. Després, Application of an ultra weak variational formulation of elliptic PDEs to the two-dimensional Helmholtz equation, SIAM J. Numer. Anal. 35 (1998) 255-299.

[8] O. Cessenat, B. Després, Using plane waves as base functions for solving time harmonic equations with the ultra weak variational formulation, J. Comput. Acoust. 11 (2003) 227-238.

[9] S.N. Chandler-Wilde, P. Monk, Wave-number-explicit bounds in timeharmonic scattering, SIAM J. Math. Anal. 39 (2008) 1428-1455.

[10] D. Colton, R. Kress, Inverse Acoustic and Electromagnetic Scattering Theory, volume 93 of Applied Mathematical Sciences, Springer, Heidelberg, 2nd edition, 1998. 
[11] P. Cummings, X. Feng, Sharp regularity coefficient estimates for complex-valued acoustic and elastic Helmholtz equations, Math. Models Methods Appl. Sci. 16 (2006) 139-160.

[12] S. Esterhazy, J. Melenk, On stability of discretizations of the Helmholtz equation, in: I. Graham, T. Hou, O. Lakkis, R. Scheichl (Eds.), Numerical Analysis of Multiscale Problems, volume 83 of Lecture Notes in Computational Science and Engineering, Springer Verlag, 2011, pp. $285-324$.

[13] X.B. Feng, H.J. Wu, $h p$-Discontinuous Galerkin methods for the Helmholtz equation with large wave number, Math. Comp. 80 (2011) 1997-2024.

[14] C.J. Gittelson, R. Hiptmair, I. Perugia, Plane wave discontinuous Galerkin methods: analysis of the $h$-version, M2AN Math. Model. Numer. Anal. 43 (2009) 297-332.

[15] U. Hetmaniuk, Stability estimates for a class of Helmholtz problems, Commun. Math. Sci. 5 (2007) 665-678.

[16] R. Hiptmair, A. Moiola, I. Perugia, Plane wave discontinuous Galerkin methods for the 2D Helmholtz equation: analysis of the $p$-version, SIAM J. Numer. Anal. 49 (2011) 264-284.

[17] R. Hiptmair, A. Moiola, I. Perugia, Stability results for the timeharmonic Maxwell equations with impedance boundary conditions, Math. Models Methods Appl. Sci. 21 (2011) 2263-2287.

[18] R. Hiptmair, A. Moiola, I. Perugia, Error analysis of Trefftz-discontinuous Galerkin methods for the time-harmonic Maxwell equations, Math. Comp. 82 (2013) 247-268.

[19] T. Huttunen, P. Monk, J.P. Kaipio, Computational aspects of the ultraweak variational formulation, J. Comput. Phys. 182 (2002) 27-46.

[20] W. McLean, Strongly elliptic systems and boundary integral equations, Cambridge University Press, Cambridge, 2000.

[21] J.M. Melenk, On Generalized Finite Element Methods, Ph.D. thesis, University of Maryland, 1995. 
[22] J.M. Melenk, On approximation in meshless methods, in: Frontiers of numerical analysis, Universitext, Springer, Berlin, 2005, pp. 65-141.

[23] J.M. Melenk, A. Parsania, S. Sauter, Generalized DG-Methods for Highly Indefinite Helmholtz Problems based on the Ultra-Weak Variational Formulation, ASC Report No. 06/2012, TU Wien, 2012.

[24] J.M. Melenk, S. Sauter, Wavenumber explicit convergence analysis for Galerkin discretizations of the Helmholtz equation, SIAM J. Numer. Anal. 49 (2011) 1210-1243.

[25] A. Moiola, Trefftz-discontinuous Galerkin methods for time-harmonic wave problems, Ph.D. thesis, Seminar for applied mathematics, ETH Zürich, 2011.

Available at http://e-collection.library.ethz.ch/view/eth: 4515.

[26] A. Moiola, R. Hiptmair, I. Perugia, Plane wave approximation of homogeneous Helmholtz solutions, Z. Angew. Math. Phys. 62 (2011) 809-837.

[27] A. Moiola, R. Hiptmair, I. Perugia, Vekua theory for the Helmholtz operator, Z. Angew. Math. Phys. 62 (2011) 779-807.

[28] P. Monk, Finite element methods for Maxwell's equations, Numerical Mathematics and Scientific Computation, Oxford University Press, 2003.

[29] P. Monk, D. Wang, A least squares method for the Helmholtz equation, Comput. Methods Appl. Mech. Eng. 175 (1999) 121-136.

[30] J. Nečas, Les méthodes directes en théorie des équations elliptiques, Masson et Cie, Éditeurs, Paris, 1967.

[31] J.C. Nédélec, Acoustic and Electromagnetic Equations: Integral Representations for Harmonic Problems, volume 44 of Applied Mathematical Sciences, Springer-Verlag, Berlin, 2001.

[32] B. Pluymers, B. van Hal, D. Vandepitte, W. Desmet, Trefftz- based methods for time-harmonic acoustics, Arch. Comput. Methods Eng. 14 (2007) 343-381.

[33] F. Rellich, Darstellung der Eigenwerte von $\Delta u+\lambda u=0$ durch ein Randintegral, Math. Z. 46 (1940) 635-636. 
[34] D. Schötzau, C. Schwab, T. Wihler, hp-dGFEM for second-order elliptic problems in polyhedra. I: Stability and quasioptimality on geometric meshes, Technical report 2009-28, SAM-ETH Zürich, 2009.

[35] D. Schötzau, C. Schwab, T. Wihler, hp-dGFEM for second-order elliptic problems in polyhedra. II: Exponential convergence, Technical report 2009-29, SAM-ETH Zürich, 2009.

[36] C. Schwab, $p$ - and $h p$-Finite Element Methods. Theory and Applications in Solid and Fluid Mechanics, Numerical Mathematics and Scientific Computation, Clarendon Press, Oxford, 1998.

[37] E.A. Spence, S.N. Chandler-Wilde, I.G. Graham, V.P. Smyshlyaev, A new frequency-uniform coercive boundary integral equation for acoustic scattering, Comm. Pure Appl. Math. 64 (2011) 1384-1415. 